\title{
Pseudo-Brewster Angular Conditions for thin Absorbing Layer on the Transparent Substrate
}

\author{
Kosobutskyy P.S. \\ Department of Computer-Aided Design \\ Lviv Polytechnic National University \\ Lviv, Ukraine.
}

\author{
Kushnir O.P. \\ Department of Physics \\ Lviv National Agrarian University \\ Dubljany, Lviv reg., Ukraine. \\ oleg.p.kushnir@gmail.com
}

\begin{abstract}
The approximate pseudo-Brewster conditions for the absorbing layer on the transparent substrate in the case when imaginary part of the phase thickness of the layer takes sufficiently small magnitudes have been obtained
\end{abstract}

Index Terms-Pseudo-Brewster angle; Envelope functions; Single-layer structure

\section{INTRODUCTION}

Pseudo-Brewster angular conditions for a single-layer transparent structure were considered earlier [1, 2]. It was shown that minima of the reflectance for $p$ - or $s$-polarization reach zero at certain angles of incidence for a single-layer structure. These angles are analogous to the well-known Brewster angle and, therefore, were called pseudo-Brewster angles.

Pseudo-Brewster angles can exist for both polarizations for single-layer plane-parallel structures unlike Brewster angle, which was introduced as the angle at which the reflectance of the interface of two media for $p$-polarized light is zero. Equality of an angle of incidence to a pseudo-Brewster angle for these structures is a necessary but insufficient requirement for achievement of zero reflectance at the certain polarization of light. For zero reflectance, it is also necessary that the phase thickness of the plane-parallel layer at this angle of incidence has been definitely chosen [2]. Thus, the pseudo-Brewster angular conditions at which zero of the reflectance is attained for a given polarization are reduced to the fulfillment of two necessary conditions: 1) the angle of incidence is equal to the pseudo- Brewster angle; 2) the phase thickness of the layer is reaching a certain magnitude, which depends on this angle.

In this paper, an attempt is made to determine the approximate pseudo-Brewster conditions for an absorbing layer on a transparent substrate in the case when the imaginary part of the phase thickness of the layer takes sufficiently small magnitudes.

\section{PSEUDO-BREWSTER ANGULAR CONDITIONS}

The envelope functions for single-layer absorbing structures can be written as [3]:

$$
R^{\max , \min }=\left(\frac{\sigma_{0,1} \pm \sigma_{1,2} \Omega}{1 \pm \sigma_{0,1} \sigma_{1,2} \Omega}\right)^{2}
$$

where $\Omega=\exp (\operatorname{Im} 2 \tilde{\delta}), \tilde{\delta}=\frac{2 \pi d}{\lambda} \sqrt{\tilde{n}_{1}^{2}-n_{0}^{2} \sin ^{2} \alpha}-$ phase thickness of a layer with thickness $d$ and complex refractive index $\tilde{n}_{1}=n_{1}-i \chi_{1}$. As follows from (1), the envelope of the minima assumes a minimum $\left(R^{\min }=0\right)$ with the condition

$$
\sigma_{0,1}=\sigma_{1,2} \Omega
$$

The solution of equation (2) is the pseudo-Brewster angle $\alpha_{p B}$, which is an analogue of the Brewster angle for a single interface between two media and is not forbidden for $p$ - and $s$ polarizations.

For the structure consisting of the absorbing film deposited on the transparent substrate, the magnitude of the pseudoBrewster angles can be determined approximately for the case when $\Omega \approx 1$, using the following expressions:

$$
\begin{gathered}
\alpha_{p B 1,2,3,4} \cong \alpha_{p B 1,2,3,4}^{0}+c\left(\alpha_{p B 1,2,3,4}^{0}\right) \cdot \operatorname{Im} \tilde{\delta}\left(\alpha_{p B 1,2,3,4}\right), \\
c\left(\alpha_{p B 1,2,3,4}^{0}\right)=\frac{2 \sigma_{1,2}\left(\alpha_{p B 1,2,3,4}^{0}\right)}{\sigma_{0,1}^{\prime}\left(\alpha_{p B 1,2,3,4}^{0}\right)-\sigma_{1,2}^{\prime}\left(\alpha_{p B 1,2,3,4}^{0}\right)}, \\
\alpha_{p B 1}^{0}=\arctan \left(n_{2,0}\right),
\end{gathered}
$$

$$
\alpha_{p B 2}^{0}=\arctan \sqrt{\frac{2\left(n_{1,0}^{2}+\chi_{1,0}^{2}\right)^{2} n_{2,0}^{2}\left(\left(n_{1,0}^{2}+\chi_{1,0}^{2}\right)^{2}-n_{2,0}^{2}\right)}{2 n_{2,0}^{4}\left(\left(n_{1,0}^{2}+\chi_{1,0}^{2}\right)^{2}-n_{1,0}^{2}+\chi_{1,0}^{2}\right)-\left(n_{1,0}^{2}+\chi_{1,0}^{2}\right)^{4}\left(n_{2,0}^{2}-1\right)+\sqrt{z}}},
$$




$$
\alpha_{p B 3}^{0}=\arctan \sqrt{\frac{2\left(n_{1,0}^{2}+\chi_{1,0}^{2}\right)^{2} n_{2,0}^{2}\left(\left(n_{1,0}^{2}+\chi_{1,0}^{2}\right)^{2}-n_{2,0}^{2}\right)}{2 n_{2,0}^{4}\left(\left(n_{1,0}^{2}+\chi_{1,0}^{2}\right)^{2}-n_{1,0}^{2}+\chi_{1,0}^{2}\right)-\left(n_{1,0}^{2}+\chi_{1,0}^{2}\right)^{4}\left(n_{2,0}^{2}-1\right)-\sqrt{z}}},
$$

$$
\alpha_{p B 4}^{0}=\arctan \left(\sqrt{\frac{n_{2,0}^{2}-\left(n_{1,0}^{2}+\chi_{1,0}^{2}\right)^{2}}{\left(n_{1,0}^{2}+\chi_{1,0}^{2}\right)^{2}-2\left(n_{1,0}^{2}-\chi_{1,0}^{2}\right)+1}}\right),
$$

where $\chi_{1,0}=\frac{\chi_{1}}{n_{0}}$, and the relative refractive indices $n_{1,0}=\frac{n_{1}}{n_{0}}$, $n_{2,0}=\frac{n_{2}}{n_{0}} ; \sigma_{01,12}^{\prime}-$ angular derivatives of the Fresnel reflection coefficients modules $\sigma_{01,12}$;

$$
\begin{aligned}
& z=\left(n_{1,0}^{2}+\chi_{1,0}^{2}\right)^{8}\left(1-n_{2,0}^{2}\right)^{2}+4\left(n_{1,0}^{2}+\chi_{1,0}^{2}\right)^{6} n_{2,0}^{4} \\
& +4\left(n_{2,0}^{2}+\chi_{1,0}^{2}\right)^{4} n_{2,0}^{4}\left(n_{2,0}^{2}-\left(n_{1,0}^{2}-\chi_{1,0}^{2}\right)\left(n_{2,0}^{2}+1\right)\right)-16 n_{1,0}^{2} \chi_{1,0}^{2} n_{2,0}^{8} .
\end{aligned}
$$

Pseudo-Brewster angles $\alpha_{p B 1,2,3}(5)$-(7) are possible only in $p$-polarization, and the pseudo-Brewster angle $\alpha_{p B 4}(8)$ is only in $s$-polarization.

Condition (2) is satisfied at angles of incidence close to pseudo-Brewster angles $\left(\alpha \rightarrow \alpha_{p B}\right)$ (3) and the envelope of minima (1) reaches magnitude close to zero $\left(R^{\min } \rightarrow 0\right)$. For these angles, the reflectance $R$

$$
R=\frac{R^{\min }+b^{2} \cos ^{2} \frac{2 \operatorname{Re} \tilde{\delta}+\phi_{01}-\phi_{12}}{2}}{1+b^{2} \cos ^{2} \frac{2 \operatorname{Re} \tilde{\delta}-\phi_{01}-\phi_{12}}{2}}, b^{2}=\frac{4 \sigma_{01} \sigma_{12} \Omega}{\left(1-\sigma_{01} \sigma_{12} \Omega\right)^{2}}
$$

is not close to zero for arbitrary wavelengths.

The envelope of the minima and the reflectance will tend to zero ( $R^{\min } \rightarrow 0, R \rightarrow 0$ ) only for the case when two conditions are simultaneously fulfilled:

1) the angle of incidence is equal to the pseudo-Brewster angle $\alpha_{p B}$;

2) the phase thickness of the layer $\tilde{\delta}\left(\alpha_{p B}\right)$ at this angle is $\tilde{\delta}_{k}\left(\alpha_{p B}\right)(k=1,2, \ldots)$,

where

$$
\begin{aligned}
& \operatorname{Re} \delta_{k}\left(\alpha_{p B}\right)=\frac{\phi_{12}\left(\alpha_{p B}\right)-\phi_{01}\left(\alpha_{p B}\right)+\pi(2 k+1)}{2} \\
& \operatorname{Im} \delta_{k}\left(\alpha_{p B}\right)=\operatorname{Re} \delta_{k}\left(\alpha_{p B}\right) \frac{\operatorname{Im} \sqrt{\tilde{n}_{1}^{2}-n_{0}^{2} \sin ^{2} \alpha_{p B}}}{\operatorname{Re} \sqrt{\tilde{n}_{1}^{2}-n_{0}^{2} \sin ^{2} \alpha_{p B}}} .
\end{aligned}
$$

Bearing in mind that the expressions for the phase thicknesses (9) depend on the pseudo-Brewster angle, and the latter according to (3) itself depends on the imaginary part of the phase thickness, the problem can be solved by one of the numerical methods, for example, by iteration.

To do this, in the first stage of the calculations, the magnitude of the imaginary part of the phase thickness $\operatorname{Im} \tilde{\delta}\left(\alpha_{p B}\right)$, which is included in (3), is replaced by $\operatorname{Im} \tilde{\delta}\left(\alpha_{p B}^{0}\right)$ and the phase thickness $\tilde{\delta}_{k}\left(\alpha_{p B}\right)$ is found based on (9) using a determined angle $\alpha_{p B}$. Next, the calculation cycles are repeated as many times until the desired accuracy $\alpha_{p B}$ and $\tilde{\delta}_{k}\left(\alpha_{p B}\right)$.

Thus, the pseudo-Brewster conditions for the absorbing layer on the transparent substrate include: 1) the expressions for the pseudo-Brewster angles $\alpha_{p B}$ (3), which depend not only on the refractive indices, but also on the absorption indices, on the thickness of the layer and on the wavelength;

2) expressions for the phase thickness of the layer $\tilde{\delta}_{k}\left(\alpha_{p B}\right)$.

Determined pseudo-Brewster conditions can be used only for the case of a small magnitude of the imaginary part of the phase thickness, which is realized under the condition of weak absorption in the layer or for a sufficiently thin layer in the long-wavelength range of the spectrum.

\section{REFERENCES}

[1] P.S. Kosobutskyy, O.P. Kushnir, "Regularities of manifestation of the brewster and pseudo-brewster angular conditions in spectra of light reflection from a thin transparent layer," Ukr. J. Phys., vol. 52, pp. 225228, 2007.

[2] P.S. Kosobutskyy, O.P. Kushnir, "Regularities of Oblique Light Reflection by a Film, Caused by a Multibeam Interference," Journal of Physical Studies, vol. 12, pp. 1701_1-1701_5, 2008.

[3] P.S. Kosobutskyy, A. Morgulis, "Simulation of three-layer transparent structures by the method of enveloping amplitude-phase fabry_perot spectra upon the normal incidence of a light beam on the interfaces," Ukr. J. Phys., vol. 49, pp. 1163-1166, 2004. 